\title{
Discontinuous Galerkin for Diffusion ${ }^{1}$ Bram van Leer ${ }^{2}$ and Shohei Nomura ${ }^{3}$
}

\author{
Department of Aerospace Engineering \\ University of Michigan \\ bram@umich.edu, snomura@umich.edu
}

\begin{abstract}
The Discontinuous Galerkin method for the diffusion equation is reformulated using a smooth locally recovered solution that in the weak sense is indistinguishable from the discontinuous discrete solution. The recovery principle creates schemes that are not included in the family of traditional DG diffusion schemes, and are potentially more accurate. A way is presented to extend the family so that the recovery-based schemes are included. It is argued that the recovery approach for the diffusive fluxes does not contradict the upwind approach for advective fluxes.

An eigenvalue/eigenvector analysis suggests that the order of accuracy of the recovery schemes is at least $2 p+2$, where $p$ is the order of the basis function. This conclusion is supported by $1-\mathrm{D}$ numerical tests, in which the new and old schemes are pitted against each other.
\end{abstract}

\section{Introduction}

Discontinuous Galerkin (DG) methods are on their way to becoming the preferred numerical tools in fluid dynamics [1,2], with significant research support from the aeronautical community $[3,4]$. They combine the flexibility of Godunov-type finite-volume methods, which employ a discontinuous solution representation, with the compactness, versatility and potential accuracy of finite-element methods.

The link with finite-volume methods is not accidental: DG methods are the finite-element analyst's answer to finite-volume methods. Dropping the requirement of continuity of basis/test functions at element boundaries changes the solution representation from global to local; this makes the method better suited for hyperbolic problems, the solutions of which often include strongly localized features such as shock waves, slip surfaces and contact discontinuties.

The first applications of DG were indeed to the advection equation (Reed and Hill(1973)[5] for the steady operator, Van Leer (1977)[6] for the unsteady operator), but soon attempts were made to apply DG to the diffusion operator. These were unsatisfactory because of a lack of numerical stabilty. One early successful DG discretization of an elliptic operator is due to Arnold (1982)[7], who introduced an interior penalty in order to penalize the discontinuities in the discrete solution.

\footnotetext{
${ }^{1}(\mathrm{C} 2005$ by Bram van Leer and Shohei Nomura. Published by the American Institute of Aeronautics and Astronautics, Inc., with permission.

${ }^{2}$ Professor, Fellow AIAA

${ }^{3}$ Graduate student, member AIAA
} 
Much more recently, Baumann (1997) $[8,9]$ ) developed a DG method that does not require the penalty term for stabilty if the order of the method is high enough. Baumann's method has the odd property that the discretization of the diffusion operator may become dispersive for high-frequency modes.

In the PhD thesis of Van Raalte [10], the Arnold and Baumann DG methods for the diffusion or Poisson equation are embedded in a two-parameter family. The first parameter, $\mu$, is the coefficient of the penalty term mentioned above; the second parameter, $\sigma$, multiplies another jump-related term, the purpose of which is to restore continuity in a weak sense. Baumann's scheme is generated by a special value of $\sigma$. Although both terms fit into a theoretical mathematical framework, further physical or numerical justification is largely lacking, nor is it clear why other, similar terms are not included.

Still other DG methods for the diffusion operator can be found in the recent literature. Arnold et al.[11] have managed to formally include all of these in a multiparameter family, but the implied relations among the schemes seem contrived, and do not bring physical insight. This currently appears to represent the state of the art in DG for diffusion.

In this paper we present a natural way of generating stable and accurate DG discretizations for the diffusion operator. Its basis is to actually remove the discontinuities, through the recovery of a smooth solution that in the weak sense is indistinguishable from the piecewise continuous discrete solution. Before getting into the details of the method (Section 4) we must re-examine the reason for using discontinuous basis functions (Section 2), which naturally arise in finitevolume advection schemes, and how this choice affects diffusion schemes (Section 3). An analysis of the evolutionary errors of both old and new schemes is found dispersed throughout Sections 3 and 4, while the analysis of the initial projection errors has been moved to Appendix A. The question whether there is a mapping between the new and old schemes is addressed in Section 5. Section 6 describes a numerical competition between the new and old schemes, based on a 1-D diffusion test case, confirming the findings of the accuracy analysis. The paper ends with an assessment of the potential impact of the present methodology, and speculations regarding the future of DG methods (Section 7).

\section{Advection and discontinuous basis functions}

\subsection{Finite-difference approach}

Consider the 1-D advection equation,

$$
u_{t}+a u_{x}=0,
$$

in which the advection speed $a$ may be positive or negative. The most simple-minded finitedifference scheme for this equation is "Forward Time, Central Space", or

$$
u_{j}^{n+1}=u_{j}^{n}-\frac{\nu}{2}\left(u_{j+1}^{n}-u_{j-1}^{n}\right),
$$

where $u_{j}^{n} \equiv u(j \Delta x, n \Delta t)$ and $\nu=a \Delta t / \Delta x$ is the Courant-Friedrichs-Lewy (CFL) number; a Von-Neumann analysis reveals this scheme is unstable. Stability can be achieved, for $|\nu| \leq 1$, by centering the scheme in time,

$$
u_{j}^{n+2}=u_{j}^{n}-\nu\left(u_{j+1}^{n+1}-u_{j-1}^{n+1}\right),
$$


or by approximating the spatial derivative on the upwind interval,

$$
u_{j}^{n+1}=u_{j}^{n}+\nu\left(u_{j}^{n}-u_{j-1}^{n}\right)
$$

here $a$ has been taken positive for the sake of determinacy. This scheme is stable for

$$
0 \leq \nu \leq 1
$$

The upwind choice can be justified by the Backward Method of Characteristics, which tells us that the solution of the advection equation can be written as

$$
u_{j}^{n+1}=u\left(x_{j}-\nu \Delta x, t^{n}\right) .
$$

Since $x_{j}-\nu \Delta x$ in general is not a grid-point, the local initial value has to be obtained by interpolation. Linear interpolation between the adjacent nodal-point values yields

$$
u\left(x_{j}, t^{n+1}\right)=(1-\nu) u_{j}^{n}+\nu u_{j-1}^{n},
$$

which is seen to be identical to Eqn. (4).

\section{$2.2 \quad$ Finite-volume approach}

When converting this scheme into a finite-volume scheme, discontinuous basis functions naturally arise. Assume that per cell we allow only one piece of information about the subcell initialvalue distribution, then conservation requires that this quantity be the average value. In what follows, integer subscripts indicate cells as well as cell centers, half-integers indicate cell faces, viz., $x_{j+\frac{1}{2}}=\left(j+\frac{1}{2}\right) \Delta x$, and $I_{j}$ is the open interval $\left(x_{j-\frac{1}{2}}, x_{j+\frac{1}{2}}\right)$, or cell interior. With this notation the global distribution can be written as

$$
u\left(x, t^{n}\right)=\bar{u}_{j}^{n}, \quad x \in I_{j}, \quad \forall j
$$

it is piecewise (cellwise) constant. Advecting this distribution over a time-interval $\Delta t$ yields the exact solution

$$
u_{e x}\left(x, t^{n+1}\right)=\bar{u}_{j}^{n}, \quad x_{j-\frac{1}{2}}+\nu \Delta x<x<x_{j+\frac{1}{2}}+\nu \Delta x, \quad \forall j .
$$

For non-integer $\nu$ this means the discontinuities have moved into the cells, creating subcell distributions governed by three quantities: $\bar{u}_{j}^{n}, \bar{u}_{j-1}^{n}$ and $\nu$. Before the next time-step can be taken, $u_{e x}\left(x, t^{n+1}\right)$ must be projected back onto the space of cellwise constant functions, which is a fancy way of saying that the subcell distributions must be made uniform again. Conservation requires that the average value must be preserved. The updated subcell distribution thus becomes

$$
u\left(x, t^{n+1}\right)=\bar{u}_{j}^{n+1}, \quad x \in I_{j}, \quad \forall j,
$$

where $\bar{u}_{j}^{n+1}$ follows from conservation:

$$
\bar{u}_{j}^{n+1}=(1-\nu) \bar{u}_{j}^{n}+\nu \bar{u}_{j-1}^{n} .
$$

It is seen that this is the same operation as in Eqn. (7), applied to cell averages rather than point values. 
This result generalizes as follows:

A finite-difference advection scheme based on $p^{\text {th }}$-order polynomial interpolation of point values of the solution maps into a finite-volume scheme based on subcell polynomial distributions of order $p-1$, and vice versa.

Furthermore, if the interpolation yielding the subcell distribution is directionally unbiased, the advection operator comes out upwind biased.

For example, the second-order upwind-biased advection scheme of Fromm (1967) [12] corresponds to a finite-volume advection scheme in which the subcell distributions are linear; the gradient in each cell is computed by central differencing of neighboring cell-averages [6]. Staying with the same stencil, the unique third-order upwind-biased advection scheme corresponds to a finite-volume scheme with quadratic subcell distributions; the second derivative used in the subcell interpolation is again obtained by central differencing.

\subsection{Discontinuous Galerkin approach}

Now the step to DG is easy to make. If one chooses to determine the parameters of the subcell distributions from inner products of the solution with test functions, rather than by finitedifferencing, then the scheme can be formulated as a finite-element scheme. If these test functions are the same as the basis functions used to build the distributions inside the elements, the finiteelement method is called a Galerkin method. If the basis functions are globally discontinuous, as above, we speak of a Discontinuous Galerkin method.

DG methods have two attractive properties vis-à-vis standard finite-volume methods:

1. their stencil does not increase with the order of the scheme; instead, more independent updates are needed per cell;

2. the schemes are more accurate than the solution representation would suggest, a property called superconvergence.

In 1977 [6] the first author presented advection schemes with linear and quadratic subcell distributions. Time integration for all schemes is by the exact advection operator. Schemes III (linear) and VI (quadratic) are DG schemes. The second-order scheme III is analyzed in detail; its two eigenvalues and corresponding eigenfunctions are derived. One eigenfunction accurately represents the solution and is advected with third-order accuracy, indicative of superconvergence; the other, inaccurate eigenfunction is damped out quickly. Eigenfunctions for Scheme VI were obtained by Woodward [13]; superconvergence for higher-order schemes of this type was demonstrated by Lowrie [14].

Schemes III and VI were reinvented in the weather-prediction community for simulating atmosheric advection; they go under the names of Russel and Lerner [15] and Prather [16], repectively. When trying to extend these schemes beyond advection, viz., to a nonlinear hyperbolic system like the Euler equations, the first author ran into insuperable difficulties because the exact shift operator no longer applies [17], and he abandoned the idea. It is understandable that in the current use of DG methods for hyperbolic systems the time integration is preferably decoupled from the spatial representation via the use of the method of lines (i. e., multistage/step schemes). 


\subsection{Finite elements with a fix}

It can be argued that the DG methods for hyperbolic equations, as these have evolved in the course of three decades, are not pure finite-element methods. This is most obvious when revisiting the first-order upwind advection scheme (11) based on the piecewise constant initial values (8). Only one family of basis/test functions is used; the function that is nonzero on $I_{j}$ reads

$$
v_{0}(x)= \begin{cases}0, & x<x_{j-\frac{1}{2}}, \\ 1, & x \in I_{j}, \\ 0, & x>x_{j+\frac{1}{2}} .\end{cases}
$$

When taking the inner product of the advection equation with this test function on $I_{j}$, as is customary in the finite-element technique, the result at any time (temporal superscripts suppressed) is

$$
\int_{I_{j}} u_{t} d x=\Delta x \frac{\partial \bar{u}_{j}}{\partial t}=-\left.a u\right|_{j-\frac{1}{2}, R} ^{j+\frac{1}{2}, L} ;
$$

here $R$ and $L$ indicate values on the right and left side, respectively, of the interface between two cells. Since $u$ is uniform inside cell $j$ according to (8), the right-hand side of (13) vanishes; this happens for all $j$. In other words, the weak form of the advection equation fails to capture the physics if $u$ is expressed solely in terms of the basis function $v_{0}^{4}$.

To escape this problem, the advection equation has to be visited once more. The nature of advection implies that the values initially at $x_{j-\frac{1}{2}, R}$ and $x_{j+\frac{1}{2}, L}$ are of no significance: an instant after $t^{n}$ the upwind value of $u$ establishes itself at each interface. For forward advection we have

$$
\begin{aligned}
& u\left(x_{j-\frac{1}{2}, R}, t^{n}+\right)=\bar{u}_{j-1}^{n}, \\
& u\left(x_{j+\frac{1}{2}, L}, t^{n}+\right)=\bar{u}_{j}^{n},
\end{aligned}
$$

and this remains so for any finite time-interval $<\Delta x / a$. Hence, the DG community has been using Riemann solvers to obtain upwind fluxes at cell interfaces.

In my opinion, though, this fix violates the basic finite-element doctrine, which asserts that satisfying the equations in the weak sense for all possible basis functions suffices to capture the physics of the equations. Apparently this is not so with discontinuous basis/test functions; thus the DG community had to borrow from the finite-volume community.

Unfortunately, the problem gets worse when combining discontinuous basis functions with the diffusion operator. Here the finite-volume community itself has failed to come up with a selfconsistent approach, so the DG community is clueless. This is the subject of the next section.

\section{Diffusion and discontinuous basis functions}

\subsection{Finite-difference approach}

Consider now the 1-D diffusion equation,

$$
u_{t}=D u_{x x}
$$

\footnotetext{
${ }^{4}$ Things do get better with higher-order representations, but these produce unstable schemes.
} 
where $D$ is the positive diffusion coefficient. The "Forward Time, Central Space" diffusion scheme,

$$
u_{j}^{n+1}=u_{j}^{n}+r\left(u_{j+1}^{n}-2 u_{j}^{n}+u_{j-1}^{n}\right),
$$

with $r=D \Delta t /(\Delta x)^{2}$, unlike its advective sister, is a perfectly fine scheme, stable for $0 \leq r \leq \frac{1}{2}$. On the other hand, centering the scheme in time, after the example of CFD pioneer Richardson (1910) [18],

$$
u_{j}^{n+2}=u_{j}^{n}+2 r\left(u_{j+1}^{n+1}-2 u_{j}^{n+1}+u_{j-1}^{n+1}\right),
$$

makes it unconditionally unstable; so does directional bias, for example,

$$
u_{j}^{n+1}=u_{j}^{n}+r\left(u_{j}^{n}-2 u_{j-1}^{n}+u_{j-2}^{n}\right) .
$$

The latter scheme is also dispersive, which by itself already is not a desirable property for a diffusion scheme ${ }^{5}$

The above examples show that, numerically speaking, the advection and diffusion operators are opposites: what is good for the goose is not good for the gander, and vice versa. We stress this because it suggests that, if a unified treatment of advection and diffusion terms can be found, it may on the surface not look unified at all.

\subsection{Finite-volume approach}

The finite-volume approach to the diffusion is pragmatic: it is inconsistent with the advection approach, but it works. In essence it is the finite-difference approach. The inconsistency is glaring in schemes for the advection-diffusion equation, itself a model for the Navier-Stokes equations,

$$
u_{t}+a u_{x}=D u_{x x}
$$

Consider, for instance, the simplest useful finite-volume advection-diffusion scheme,

$$
\bar{u}_{j}^{n+1}=\bar{u}_{j}^{n}-\nu\left(\bar{u}_{j}^{n}-\bar{u}_{j-1}^{n}\right)+r\left(\bar{u}_{j+1}^{n}-2 \bar{u}_{j}^{n}+\bar{u}_{j-1}^{n}\right) .
$$

Here the advection and diffusion terms are based on different inital-value interpretations. For the advection term the discontinuous piecewise uniform distribution (8) and knowledge of the flow direction are used, while in the diffusion term it seems the cell averages are treated as point values. If one wished to come up with a global initial-value distribution from which the diffusion term could be derived, it would be the continuous piecewise linear distribution

$$
\mathcal{U}\left(x, t^{n}\right)=\frac{1}{2}\left(\bar{u}_{j}^{n}+\bar{u}_{j+1}^{n}\right)+\left(x-x_{j+\frac{1}{2}}\right) \frac{\bar{u}_{j+1}^{n}-\bar{u}_{j}^{n}}{\Delta x}, \quad x \in\left[x_{j}, x_{j+1}\right], \quad \forall j .
$$

\footnotetext{
${ }^{5}$ This may seem unfair to diffusion schemes: if an advection scheme like (4) can be dissipative, why can't a diffusion scheme be dispersive? Conversely, if dispersive errors are avoidable in diffusion schemes, why not insist on avoiding dissipative errors in advection schemes? The flaw in this reasoning lies in the asymmetry of advection and diffusion in relation to numerical stability. Nondissipative advection schemes like the leap-frog scheme (3) necessarily are only neutrally stable. Some dissipation is needed in the nonlinear case to increase robustness, and for handling shock waves. In addition, disspation can be helpful in damping waves that are badly out of step because of dispersive errors.
} 
Note that this interpolation does not conserve the cell averages on the original grid $\left(\overline{\mathcal{U}}_{j}^{n} \neq \bar{u}_{j}\right)$, although it does so on the staggered grid.

This is the example after which the inviscid and viscous/conductive terms in finite-volume Navier-Stokes codes are patterned. The inviscid fluxes are computed with higher-order accuracy, using linear or quadratic subcell interpolation combined with a Riemann solver; the computation of the viscous/conductive fluxes at a cell face is based on Gauss's integral theorem:

$$
(\nabla u)_{f a c e}=\frac{1}{V} \oint_{S_{f a c e}} u \hat{\mathbf{n}} d S
$$

Here the surface $S_{\text {face }}$ surrounds the cell face; $V$ is the volume inside $S_{\text {face }}$. The surface runs through the centers of the cells neighboring the face; the integral is usually computed in a cavalier fashion, e. g., as if the distributions of $u$ in those cells were uniform. In one dimension Eqn. (23) reduces to

$$
\left(u_{x}\right)_{j+\frac{1}{2}}=\frac{\bar{u}_{j+1}^{n}-\bar{u}_{j}^{n}}{\Delta x}
$$

consistent with Eqn. (22). For future reference we repeat below the simplest finite-volume scheme for pure diffusion, i. e., Eqn. (21) with $\nu=0$,

$$
\bar{u}_{j}^{n+1}=\bar{u}_{j}^{n}+r\left(\bar{u}_{j+1}^{n}-2 \bar{u}_{j}^{n}+\bar{u}_{j-1}^{n}\right),
$$

stable for $0 \leq r \leq \frac{1}{2}$.

A unified treatment of advection and diffusion based on the discontinuous initial values (8) might require, so it seems, a short-time exact solution of the diffusion equation at each jump, with the smoothed distribution advecting as a whole. This procedure, however, does not yield a consistent approximation of the advection-diffusion operator. Specifically, for decreasing timesteps the time-averaged diffusive fluxes are unbounded, behaving as $(D \Delta t)^{-\frac{1}{2}}$; in addition, the instantaneous smoothing changes the upwind interface values back into face-centered averages. Again it appears that what is good for advection, i. e., using an exact short-term solution to get the interface flux, is not good for diffusion.

\subsection{Discontinuous Galerkin approach}

In the absence of a solid finite-volume framework, finite-element specialists have been at a loss regarding the approximation of the diffusion operator with discontinuous basis functions. In consequence, ad hoc terms have been introduced into the schemes based on the theory of Lagrange multipliers; see, e. g., [10]. All are bilinear in $(u, v)$ and consistent with the diffusion equation in the weak sense, i. e., a solution of the original PDE satisfies the discrete equations; further justification, physical or numerical, is largely lacking. Moreover, it is not clear why certain bilinear forms are selected while other ones are not.

To understand the action of these terms on a cellwise basis, take the inner product of the 1-D diffusion equation with any discontinuous test function $v(x)$ that is nonzero only on the open interval $I_{j}$; using partial integration on the right-hand side this yields

$$
\int_{I_{j}} v u_{t} d x=v u_{x} \mid \begin{aligned}
& j+\frac{1}{2}, L \\
& j-\frac{1}{2}, R
\end{aligned}-\int_{I_{j}} v_{x} u_{x} d x
$$


According to this equation, the solution in cell $j$ is independent of the solution in its neighbors, which is unacceptable. Unlike for the advection operator, there is no physically obvious way to introduce coupling between the cells. Enter the finite-element interface terms.

To begin with, the right-hand side of Eqn. (26) is expanded with terms originating in the adjacent cells; these equal zero because $v$ vanishes outside cell $j$,

$$
\int_{I_{j}} v u_{t} d x=-D\left(\left.\left[v u_{x}\right]\right|_{j+\frac{1}{2}}+\left.\left[v u_{x}\right]\right|_{j-\frac{1}{2}}\right)-D \int_{I_{j}} v_{x} u_{x} d x
$$

Here the square brackets indicate a jump across an interface,

$$
[q]_{j+\frac{1}{2}}=q_{j+\frac{1}{2}, R}-q_{j+\frac{1}{2}, L}
$$

in what follows we shall also use the average across an interface,

$$
<q>_{j+\frac{1}{2}}=\frac{1}{2}\left(q_{j+\frac{1}{2}, L}+q_{j+\frac{1}{2}, R}\right)
$$

Using this notation the jumps in Eqn. (27) can be expanded:

$$
\begin{aligned}
\int_{I_{j}} v u_{t} d x & =-D\left\{\left.\left(<v>\left[u_{x}\right]+<u_{x}>[v]\right)\right|_{j+\frac{1}{2}}\right. \\
& \left.+\left.\left(<v>\left[u_{x}\right]+<u_{x}>[v]\right)\right|_{j-\frac{1}{2}}\right\}-D \int_{I_{j}} v_{x} u_{x} d x
\end{aligned}
$$

This equation is still only a rewrite of Eqn. (26).

Now the coupling between the cells is established by dropping the contributions from $\langle v\rangle$ $\left[u_{x}\right]$. The motivation for keeping the other contribution, $\left\langle u_{x}\right\rangle[v]$, is that it is proportional to the average diffusive flux at the interface, which is considered valuable in keeping the scheme conservative. The resulting scheme,

$$
\int_{I_{j}} v u_{t} d x=-D\left(<u_{x}>\left.[v]\right|_{j+\frac{1}{2}}+<u_{x}>\left.[v]\right|_{j-\frac{1}{2}}\right)-D \int_{I_{j}} v_{x} u_{x} d x
$$

indeed is conservative (i. e., in flux form) with regard to $\int_{I_{j}} u d x$, as can be checked by inserting $v=v_{0}$ from (12).

To increase the chances of coming up with stable and accurate diffusion schemes, two more bilinear interface terms are added:

$$
\begin{aligned}
\int_{I_{j}} v u_{t} d x & =-D\left(<u_{x}>\left.[v]\right|_{j+\frac{1}{2}}+<u_{x}>\left.[v]\right|_{j-\frac{1}{2}}\right)-D \int_{I_{j}} v_{x} u_{x} d x \\
& +\sigma D\left(<v_{x}>\left.[u]\right|_{j+\frac{1}{2}}+<v_{x}>\left.[u]\right|_{j-\frac{1}{2}}\right) \\
& -\frac{\mu D}{\Delta x}\left(\left.[v][u]\right|_{j+\frac{1}{2}}+\left.[v][u]\right|_{j-\frac{1}{2}}\right) .
\end{aligned}
$$

The term with coefficient ${ }^{6} \mu$ is the internal penalty function of Arnold [7], which penalizes the solution for being discontinuous. The coefficient must be positive for a lower-order scheme; it may

\footnotetext{
${ }^{6}$ In order to make $\mu$ nondimensional We have taken a factor $(\Delta x)^{-1}$ out of the original definition of $\mu$.
} 
be taken zero in higher-order schemes. The term with coefficient $\sigma$ is said to restore continuity in a weak sense. The coefficient $\sigma$ must be nonzero in higher-order schemes. The choice $\sigma=-1$ yields the oldest known, so-called Symmetric discretization; it needs the internal-penalty term for stability. The choice $\sigma=1$ is due to Baumann [8, 9]; it yields stable higher-order discretizations without the penalty term. It has the odd property of becoming dispersive with the use of basis functions of degree 3 or higher [10]; whether this property is really so odd is discussed in the footnote to Section 3.1.

In the next subsection we shall investigate if the above combination of terms indeed captures the physics of the diffusion operator.

\subsection{Piecewise constant solution}

The degree of the polynomials used for the subcell representation of the solution is called $p$; assume first that $p=0$. The solution $u$ is assumed to be piecewise constant as in (8), and the only test function to be applied is $v_{0}(x)$ from Eqn. (12). Hence both $v_{x}$ and $u_{x}$ vanish on $I_{j}$, which makes all terms on the right-hand side of Eqn. (32) vanish except the penalty term:

$$
\begin{aligned}
\frac{1}{\Delta x} \int_{I_{j}} u_{t} d x & =\left(\bar{u}_{j}\right)_{t} \\
& =-\frac{D \mu}{(\Delta x)^{2}}\left\{(-1)\left(\bar{u}_{j+1}-\bar{u}_{j}\right)+(+1)\left(\bar{u}_{j}-\bar{u}_{j-1}\right)\right\} \\
& =\frac{D \mu}{(\Delta x)^{2}}\left(\bar{u}_{j+1}-2 \bar{u} j+\bar{u}_{j-1}\right) .
\end{aligned}
$$

This equation is consistent with the diffusion equation (16) only for one choice of the penalty parameter $\mu$,

$$
\mu=1
$$

to find this value one needs to consult the original diffusion equation. This is another instance where the weak formulation alone does not suffice to produce a valid finite-element method; cf. Section 2.4. For future reference we repeat the resulting scheme in operator notation,

$$
\frac{\partial \bar{u}_{j}}{\partial t}=\frac{D}{(\Delta x)^{2}}\left(\bar{u}_{j+1}-2 \bar{u}_{j}+\bar{u}_{j-1}\right)
$$

\subsection{Piecewise linear solution}

Now consider the case $p=1$. In addition to $v_{0}$ a linear test function $v_{1}$ is needed; the one that does not vanish in cell $j$ is given by

$$
v_{1}(x)= \begin{cases}0, & x<x_{j-\frac{1}{2}}, \\ x-x_{j}, & x \in I_{j}, \\ 0, & x>x_{j+\frac{1}{2}}\end{cases}
$$


It has the desirable property that its integral over $I_{j}$ vanishes. Hence, when expressing the discrete solution in terms of $v_{0}$ and $v_{1}$,

$$
u(x)=\bar{u}_{j}+\left(x-x_{j}\right) \frac{\overline{\Delta u}_{j}}{\Delta x}, \quad x \in I_{j}, \quad \forall j,
$$

where $\overline{\Delta u}_{j}$ is the "undivided gradient" in cell $j$, averaging $u(x)$ over $I_{j}$ returns $\bar{u}_{j}$. This conservation property is secured to arbitrary order $p$ by making each $v_{m}$ a Legendre polynomial of degree $m$, scaled to the interval $I_{j}$ and suitably normalized [6].

To retrieve $\overline{\Delta u}_{j}$, use test function $v_{1}$ on $I_{j}$,

$$
\int_{I_{j}} v_{1} u d x=\frac{\overline{\Delta u}_{j}}{\Delta x} \int_{I_{j}} v_{1}^{2} d x
$$

yielding

$$
\overline{\Delta u}_{j}=\Delta x \frac{\int_{I_{j}}\left(x-x_{j}\right) u d x}{\int_{I_{j}}\left(x-x_{j}\right)^{2} d x}=\frac{12}{(\Delta x)^{2}} \int_{I_{j}}\left(x-x_{j}\right) u d x .
$$

Working out scheme (32) for the two test functions yields the following differential equations for updating $\bar{u}_{j}$ (this one is conservative) and $\overline{\Delta u}_{j}$ :

$$
\frac{\partial}{\partial t}\left(\begin{array}{c}
\bar{u}_{j} \\
\overline{\Delta u}_{j}
\end{array}\right)=\frac{D}{(\Delta x)^{2}} M(T, \sigma, \mu)\left(\begin{array}{c}
\bar{u}_{j} \\
\overline{\Delta u}_{j}
\end{array}\right)
$$

with

$$
M(T, \sigma, \mu)=\left(\begin{array}{cc}
\mu\left(T-2 I+T^{-1}\right) & \frac{1-\mu}{2}\left(T-T^{-1}\right) \\
6(\sigma+\mu)\left(T-T^{-1}\right) & 3\left(T-2 I+T^{-1}\right)-3(\sigma+\mu)\left(T+2 I+T^{-1}\right)
\end{array}\right)
$$

here $T$ represents forward translation by one cell,

$$
T q_{j}=q_{j+1}
$$

The eigenvalues of the matrix operator $M(T, \sigma, \mu)$ can be found through Fourier analysis. With

$$
\left(\begin{array}{c}
\bar{u}_{j} \\
\overline{\Delta u}_{j}
\end{array}\right)=e^{i \beta j}\left(\begin{array}{c}
\bar{u}_{0} \\
\overline{\Delta u}_{0}
\end{array}\right)
$$

the operator $T$ reduces to scalar multiplication,

$$
\hat{T}=e^{i \beta}
$$

the following Fourier symbols are frequently used in this and further sections:

$$
\begin{aligned}
\hat{T}-\hat{T}^{-1} & =2 i \sin \beta, \\
\hat{T}-2 \hat{I}+\hat{T}^{-1} & =-2(1-\cos \beta), \\
\hat{T}+2 \hat{I}+\hat{T}^{-1} & =2(1+\cos \beta) .
\end{aligned}
$$


For the matrix operator $M(T, \sigma, \mu)$ given by Eqn. (41) the following Fourier transform is found:

$$
\hat{M}(\beta, \sigma, \mu)=\left(\begin{array}{cc}
-2 \mu(1-\cos \beta) & i(1-\mu) \sin \beta \\
12 i(\sigma+\mu) \sin \beta & -6(1+\sigma+\mu)+6(1-\sigma-\mu) \cos \beta
\end{array}\right) .
$$

This matrix is an approximation of the exact differential operator; since the Fourier symbol of spatial differentiation is

$$
\frac{\hat{\partial}}{\partial x}=\frac{i \beta}{\Delta x}
$$

the exact operator is

$$
\hat{M}_{e x}(\beta)=-\beta^{2} I .
$$

Dropping the $\mu$-dependence for the moment, the equation for the eigenvalues of $\hat{M}$ becomes

$$
\lambda^{2}+6 \lambda[1+\sigma-(1-\sigma) \cos \beta]+12 \sigma \sin ^{2} \beta=0 .
$$

Baumann's choice $\sigma=1$ yields

$$
\lambda_{1,2}=-6\left(1 \mp \sqrt{1-\frac{1}{3} \sin ^{2} \beta}\right) .
$$

Both eigenvalues are negative, indicating stability of the DG scheme. The eigenvalue with the minus sign,

$$
\lambda_{1}=-\beta^{2}+O\left(\beta^{4}\right),
$$

represents the exact diffusion operator (50) with second-order accuracy. The other eigenvalue, which lies between -12 and $-6-2 \sqrt{6}$, corresponds to a grossly inaccurate eigenfunction, of which only an amount $O\left((\Delta x)^{4}\right)$ is present in the discretization of the Fourier mode; this is shown in Appendix A. It is fortunate that the eigenvalue lies far away from the origin; its eigenfunction may be strongly damped by a suitable time-integrator, without affecting the evolution of the accurate eigenfunction.

A peculiarity of the Baumann scheme is that the first eigenvalue vanishes for $\beta=\pi$, meaning that the Fourier mode with wavelength $2 \Delta x$ is not damped. This property may show up in the solution as odd-even grid decoupling; cf. Section 6.

Using $\sigma=-1$ yields the Symmetric scheme; for this choice the eigenvalues have opposite signs:

$$
\lambda_{1,2}=6\left(\cos \beta \mp \sqrt{1-\frac{2}{3} \sin ^{2} \beta}\right) .
$$

The "good" eigenvalue again equals $-\beta^{2}+O\left(\beta^{4}\right)$; the "bad" one varies between +12 and 0 . It is clear that stabilization of the Symmetric scheme requires inclusion of the penalty term. The lowest value of $\mu$ yielding stability is $\mu=1$; in this case $(\sigma=-1, \mu=1)$ the matrix $\hat{M}$ is diagonal, meaning that the updates of cell averages and gradients are completely independent. In consequence, the update scheme for $\bar{u}_{j}$ simply is (35), the single consistent scheme found for $p=0$. The eigenvalues (diagonal elements) are

$$
\lambda_{1}=-2(1-\cos \beta)=-\beta^{2}+O\left(\beta^{4}\right), \quad \lambda_{2}=-6(1-\cos \beta) .
$$


The first eigenvalue is just the Fourier symbol of $T-2 I+T^{-1}$. The bad eigenfunction corresponding to the second eigenvalue this time occurs with amplitude $O\left((\Delta x)^{2}\right)$ in the discretized Fourier mode; see Appendix A.

Since both the Baumann and the Stabilized Symmetric scheme are only second-order accurate, just as scheme (33-34) for $p=0$, it seems that the addition of the linear basis/test function has bought us nothing. No wonder: in neither scheme is the update equation for $\overline{\Delta u}_{j}$ consistent with the diffusion equation! One would expect the gradient update to approximate the third-order PDE

$$
\frac{\partial}{\partial t}\left(\frac{\partial u}{\partial x}\right)=D \frac{\partial^{2}}{\partial x^{2}}\left(\frac{\partial u}{\partial x}\right)
$$

Omitting details, we state here that analysis of the full $(\sigma, \mu)$-family reveals consistency is achieved only if

$$
\sigma+\mu=\frac{5}{2}
$$

Among the schemes satisfying this constraint there is one, not studied previously, that achieves fourth-order accuracy, viz., the scheme with

$$
\mu=\frac{9}{4}, \sigma=\frac{1}{4}
$$

A discussion of its properties is deferred to a future journal paper.

For further analysis of the family of schemes (32), especially with regard to their use in multigrid relaxation, the reader is referred to [10].

The switch in the sign of $\sigma$ between the Symmetric and Baumann schemes represents a dramatic change in algorithm, not usually encountered in CFD. That such a change has been considered rather recently is evidence of the lack of a guiding principle in developing DG schemes for diffusion.

The idea of making the update equation for the gradient consistent with the once-differentiated diffusion equation could be extended to update equations for higher derivatives $(p \geq 2)$, but applying it would require the availability of an ever increasing number of parameters beyond $\sigma$ and $\mu$.

In the next section we shall present a guiding principle for the use of discontinuous basis functions for diffusion, from which stable and optimally accurate DG schemes can be derived without the introduction of ad hoc terms; moreover, there is no conceptual conflict with the established treatment of advection.

\section{DG discretization of the diffusion operator}

\subsection{The recovery method}

When treating the diffusion operator with discontinuous basis functions, removing the discontinuities in a weak sense is the right idea. What has been lacking so far, not just in the DG community but also in the finite-volume community, is an operational principle. Finite-element 
specialists have tried hardest, but they appear to be ruled by their powerful computational toolbox to such a degree that they can't think "out of the box" 7

The guiding principle in removing the discontinuities is to realize that the piecewise continuous discrete solution is only a cartoon of the true solution. The first-order advection operator does not seem to mind, but the second-order diffusion operator chokes on this solution representation. What needs to be done in the latter case is to locally recover the underlying smooth solution with sufficient fidelity.

This local recovery will be used to supply information at a cell face, so it must be done in an interval bracketing the cell face. Since the available discrete data all are obtained from cellwise inner products, the interval must at least span the full cells adjacent to the face. It should not be wider either, since the whole point of DG schemes is to allow more internal basis function rather than widen the stencil. Thus, the interval is chosen as the union of the two neighboring cells; note that it is unbiased. This makes it the proper choice with regard to diffusion - and, in turn, useless for advection.

What follows is an exercise in recovery. Let us assume there initially was a smooth spatial distribution $U(x)$ that got approximated by the piecewise continuous discrete distribution $u(x)$; the desired recovered smooth distribution is called $f(x)$. Near the interface $j+\frac{1}{2}$, these functions are designed to be equal in the following weak sense:

$$
\left.\begin{array}{l}
\int_{I_{j}} v_{m} U d x=\int_{I_{j}} v_{m} u d x=\int_{I_{j}} v_{m} f d x \\
\int_{I_{j+1}} v_{m} U d x=\int_{I_{j+1}} v_{m} u d x=\int_{I_{j+1}} v_{m} f d x
\end{array}\right\}, m=0, . ., p .
$$

The basis functions $v_{m}$ are chosen to be Legendre polynomials of order up to $p \geq 0$. With the above $2 p+2$ equations, $f$ can be reconstructed as a polynomial of degree $2 p+1$, and the order of accuracy of the resulting scheme can be as high as $2 p+2$, indicating superconvergence. In fact, the analysis presented further below suggests that the order of accuracy may even be even higher, but this has not yet been demonstrated in practice.

\subsection{Example: piecewise constant basis}

We will first explore the case $p=0$ that has figured in all previous examples. The reconstruction is linear; in the interval centered on $x_{j+\frac{1}{2}}$ the function may be written in terms of the local coordinate $\xi=x-x_{j+\frac{1}{2}}$,

$$
f(\xi)=f_{0}+\xi f_{0}^{\prime}, \quad-\Delta x<\xi<\Delta x .
$$

It is likely that the original distribution $U(x)$ was not linear on this interval, but this is the best we can do. The equations (59), with $p=0$ and $v_{0}$ as in (12), become

$$
\begin{aligned}
& \frac{1}{\Delta x} \int_{-\Delta x}^{0} f d \xi=\bar{u}_{j}, \\
& \frac{1}{\Delta x} \int_{0}^{\Delta x} f d \xi=\bar{u}_{j+1},
\end{aligned}
$$

\footnotetext{
${ }^{7}$ In fact, when they do, they do not seem to recognize this. In Sections 2.4 and 3.4 two examples are given in which the weak formulation of the PDE is not enough to represent the physics, so the equation has to be consulted again. But the significance of this has gone unnoticed.
} 
from which the desired quantity can be obtained,

$$
f_{0}^{\prime}=\frac{1}{\Delta x}\left(\bar{u}_{j+1}-\bar{u}_{j}\right)
$$

The DG equations are obtained by starting from Eqn. (26) and doing one more integration by parts:

$$
\int_{I_{j}} v_{m} u_{t} d x=\left.D\left\{v_{m} f_{x}-\left(v_{m}\right)_{x} f\right\}\right|_{\mid \begin{array}{l}
j+\frac{1}{2}, L \\
j-\frac{1}{2}, R
\end{array}} ^{j}+D \int_{I_{j}}\left(v_{m}\right)_{x x} u d x, \quad m=1, \ldots, p .
$$

Note that the integral on the right-hand side only kicks in at $p=2$, and can not be further expanded. Furthermore, although a smooth solution has been recovered at each interface, the interface subscripts $L$ and $R$ are still needed because the test functions $v_{m}$ are discontinuous.

Using only $v_{0}$ yields

$$
\left(\bar{u}_{j}\right)_{t}=\frac{D}{\Delta x}\left\{\left.\left(f_{0}^{\prime}\right)\right|_{j+\frac{1}{2}}-\left.\left(f_{0}^{\prime}\right)\right|_{j-\frac{1}{2}}\right\}
$$

note that this scheme is in flux form, hence conservative. Inserting $f^{\prime}$ from (63) into (65) reproduces (35), the only consistent scheme for $p=0$, but this time for the right reason.

There is no conflict with the upwind advection discretization once we realize, as argued before, that advection and diffusion are essentially different and must exploit the given data in different ways. To drive the point home, let us recover from (60-62) the interface value of $f$,

$$
f_{0}=\frac{1}{2}\left(\bar{u}_{j}+\bar{u}_{j+1}\right)
$$

This value is not needed in the DG method for $p=0$, but diffusion afficionados might suggest we use the value to compute the advection flux figuring in Eqn. (13). This then yields the unstable central-differencing approximation that appeared earlier in (2), applied this time to cell averages rather than point values. Again there is reciprocity: what is good for the gander is not good for the goose.

In conclusion: advection and diffusion operators require different interpretations of the same data. For an advective flux we ignore the downwind data and use the discrete solution "as is" on the upwind side of the interface. For a diffusive flux we use the data from both sides, but we must locally recover a smooth solution representation. Note that for the evaluation of the diffusion term no global solution representation will be available.

\subsection{Piecewise linear basis}

Now assume the solution is piecewise linear, that is, $p=1$. For the reconstruction of $f(\xi)$ in cells $j$ and $j+1$ four data are now available, hence it can be made a cubic polynomial,

$$
f(\xi)=f_{0}+\xi f_{0}^{\prime}+\frac{\xi^{2}}{2} f_{0}^{\prime \prime}+\frac{\xi^{3}}{6} f_{0}^{\prime \prime \prime} .
$$

The equations relating $f$ to the data are (61) and (62), supplemented by the pair generated by $v_{1}$,

$$
\begin{aligned}
& \frac{12}{(\Delta x)^{2}} \int_{-\Delta x}^{0}\left(\xi+\frac{\Delta x}{2}\right) f d \xi=\overline{\Delta u}_{j} \\
& \frac{12}{(\Delta x)^{2}} \int_{0}^{\Delta x}\left(\xi-\frac{\Delta x}{2}\right) f d \xi=\overline{\Delta u}_{j+1} .
\end{aligned}
$$


Computing the integrals with (67) yields the system

$$
\left(\begin{array}{cccc}
1 & -\frac{\Delta x}{2} & \frac{(\Delta x)^{2}}{6} & -\frac{(\Delta x)^{3}}{24} \\
1 & \frac{\Delta x}{2} & \frac{(\Delta x)^{2}}{6} & \frac{(\Delta x)^{3}}{24} \\
0 & \Delta x & -\frac{(\Delta x)^{2}}{2} & \frac{3(\Delta x)^{3}}{20} \\
0 & \Delta x & \frac{(\Delta x)^{2}}{2} & \frac{3(\Delta x)^{3}}{20}
\end{array}\right)\left(\begin{array}{c}
f_{0} \\
f_{0}^{\prime} \\
f_{0}^{\prime \prime} \\
f_{0}^{\prime \prime \prime}
\end{array}\right)=\left(\begin{array}{c}
\bar{u}_{j} \\
\bar{u}_{j+1} \\
\overline{\Delta u}_{j} \\
\overline{\Delta u}_{j+1}
\end{array}\right)
$$

with solution

$$
\begin{aligned}
f_{0} & =\frac{1}{2}\left(\bar{u}_{j}+\bar{u}_{j+1}\right)-\frac{1}{6}\left(\overline{\Delta u}_{j+1}-\overline{\Delta u}_{j}\right), \\
f_{0}^{\prime} & =\frac{1}{\Delta x}\left\{\frac{9}{4}\left(\bar{u}_{j+1}-\bar{u}_{j}\right)-\frac{5}{8}\left(\overline{\Delta u}_{j}+\overline{\Delta u}_{j+1}\right)\right\}, \\
f_{0}^{\prime \prime} & =\frac{1}{(\Delta x)^{2}}\left(\overline{\Delta u}_{j+1}-\overline{\Delta u}_{j}\right) \\
f_{0}^{\prime \prime \prime} & =-\frac{15}{(\Delta x)^{3}}\left\{\bar{u}_{j+1}-\bar{u}_{j}-\frac{1}{2}\left(\overline{\Delta u}_{j}+\overline{\Delta u}_{j+1}\right)\right\} \\
& =-\frac{15}{(\Delta x)^{3}}[u]_{j+\frac{1}{2}} .
\end{aligned}
$$

Only $f_{0}$ and $f_{0}^{\prime}$ are needed in the DG method for $p=1$.

Inserting $v_{0}$ and $v_{1}$ into Eqn. (64) produces the equation pair

$$
\begin{aligned}
\left(\bar{u}_{j}\right)_{t} & =\frac{D}{\Delta x}\left\{\left.f_{0}^{\prime}\right|_{j+\frac{1}{2}}-\left.f_{0}^{\prime}\right|_{j-\frac{1}{2}}\right\} \\
\left(\overline{\Delta u}_{j}\right)_{t} & =\frac{6 D}{\Delta x}\left\{\left.f_{0}^{\prime}\right|_{j+\frac{1}{2}}-2 \frac{\left.f_{0}\right|_{j+\frac{1}{2}}-\left.f_{0}\right|_{j-\frac{1}{2}}}{\Delta x}+\left.f_{0}^{\prime}\right|_{j-\frac{1}{2}}\right\}
\end{aligned}
$$

which, combined with Eqs. (71) and (72), leads to the Recovery scheme

$$
\frac{\partial}{\partial t}\left(\begin{array}{c}
\bar{u}_{j} \\
\overline{\Delta u}_{j}
\end{array}\right)=\frac{D}{(\Delta x)^{2}}\left(\begin{array}{cc}
\frac{9}{4}\left(T-2 I+T^{-1}\right) & -\frac{5}{8}\left(T-T^{-1}\right) \\
\frac{15}{2}\left(T-T^{-1}\right) & 2\left(T-2 I+T^{-1}\right)-\frac{15}{4}\left(T+2 I+T^{-1}\right)
\end{array}\right)\left(\begin{array}{c}
\bar{u}_{j} \\
\overline{\Delta u}_{j}
\end{array}\right)
$$

The update equation for $\overline{\Delta u}_{j}$ can be shown to be consistent with the third-order PDE (56).

The Fourier transform of the matrix operator in this equation, $M_{r e c}$, becomes

$$
\hat{M}_{r e c}(\beta)=\left(\begin{array}{cc}
-\frac{9}{2}(1-\cos \beta) & -\frac{5}{4} i \sin \beta \\
15 i \sin \beta & -4(1-\cos \beta)-\frac{15}{2}(1+\cos \beta)
\end{array}\right)
$$

its eigenvalues are given by

$$
\lambda_{1,2}=-\frac{15}{2}-\frac{1}{2}(1-\cos \beta) \pm \frac{15}{2} \sqrt{1-\frac{2}{5}(1-\cos \beta)-\frac{11}{225}(1-\cos \beta)^{2}} .
$$


Both eigenvalues are negative real, making the scheme stable; the bad eigenvalue (with the minus sign) runs from -15 to -9 . Expanding the accurate eigenvalue (with the plus sign) yields

$$
\lambda_{1}=-\beta^{2}+O\left(\beta^{6}\right) .
$$

Thus, the scheme has an eigenfunction that represents the diffusion operator with fourth-order accuracy. It turns out that the bad eigenfunction, corresponding to $\lambda_{2}$, occurs only with relative amplitude $O\left((\Delta x)^{6}\right)$ in the discretized Fourier mode, so the Recovery scheme is truly fourth-order accurate; see Appendix A.

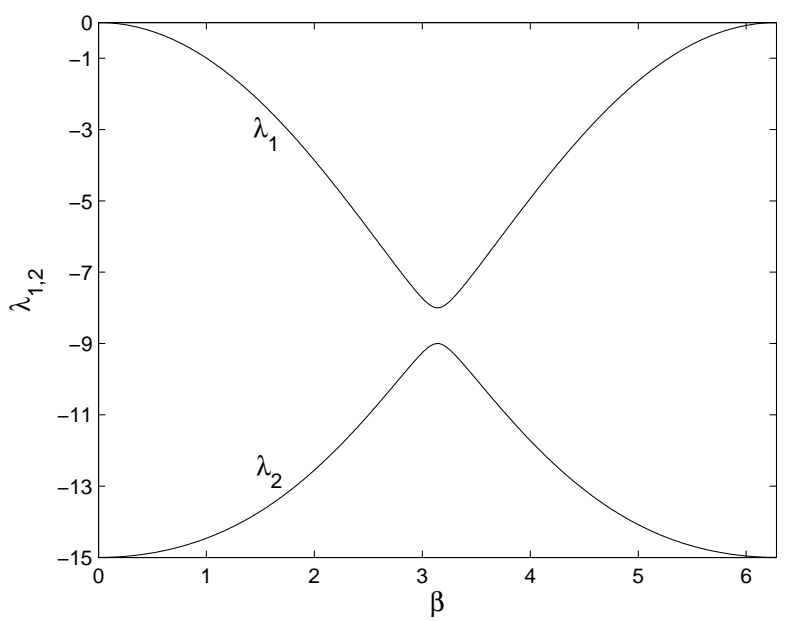

Figure 1: Eigenvalues $\lambda_{1}$ and $\lambda_{2}$ as functions of $\beta$ for the Optimal Recovery scheme with $p=1$. Note that both eigenvalues are negative, indicating the stability of the DG scheme.

Graphs of the eigenvalues as functions of $\beta$ are given in Figure 1. Figure 2 confirms the validity of Eqn. (80).

The stability of the Recovery scheme for $p=1$ and $p=2$ (see next section) is no guarantee of stability for higher values of $p$; a general proof remains to be found. If the optimal recovery (most accurate for a given $p$ ) happens to yield a stable scheme, other stable schemes of reduced accuracy can always be obtained by smoothing the recovered solution, as shown in Section 5.2.

\subsection{Piecewise quadratic basis}

Finally, assume the solution is piecewise quadratic, i.e., $p=2$. In addition to $v_{0}$ and $v_{1}$ a quadratic basis/test function is needed; the one that does not vanish in cell $j$ is given by

$$
v_{1}(x)= \begin{cases}0, & x<x_{j-\frac{1}{2}}, \\ \left(x-x_{j}\right)^{2}-\frac{(\Delta x)^{2}}{12}, & x \in I_{j}, \\ 0, & x>x_{j+\frac{1}{2}}\end{cases}
$$




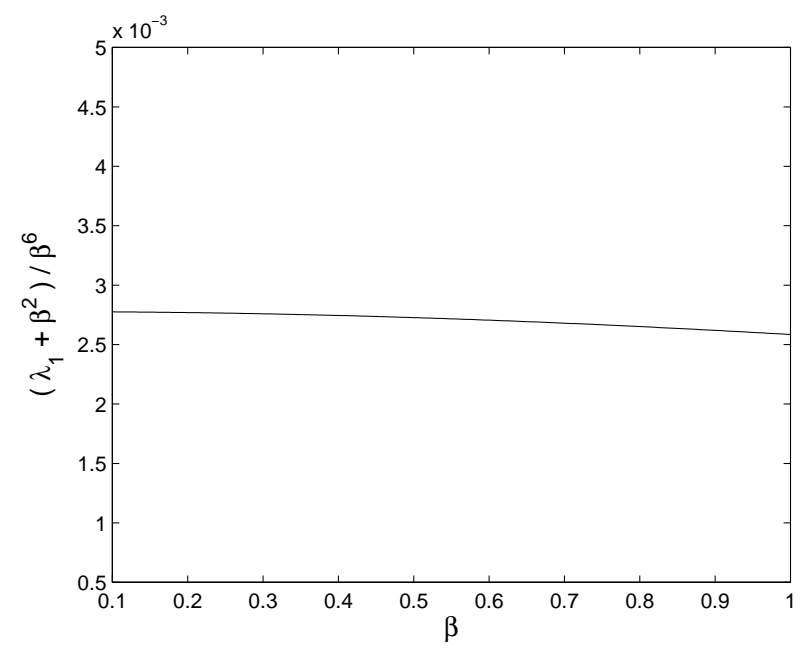

Figure 2: Error in the accurate eigenvalue $\lambda_{1}$ as a function of $\beta$, normalized by $\beta^{6}$, for the Optimal recovery scheme with $p=1$.

On $I_{j}$ the function is equivalent to the second-degree Legendre polynomial, usually given as $\frac{3}{2} x^{2}-\frac{1}{2}$ on the interval $[-1,1]$.

The representation of the solution becomes

$$
u(x)=\bar{u}_{j}+\left(x-x_{j}\right) \frac{\overline{\Delta u}_{j}}{\Delta x},+\frac{1}{2}\left\{\left(x-x_{j}\right)^{2}-\frac{(\Delta x)^{2}}{12}\right\} \frac{\bar{\Delta}^{2} u_{j}}{(\Delta x)^{2}}, \quad x \in I_{j}, \quad \forall j ;
$$

where we have introduced the undivided second derivative $\bar{\Delta}^{2} u_{j}$. Integrating this function over $I_{j}$ returns $\bar{u}_{j}$, as required by conservation. To retrieve $\bar{\Delta}^{2} u_{j}$, use test function $v_{2}$ on $I_{j}$,

$$
\int_{I_{j}} v_{2} u d x=\frac{1}{2} \frac{\bar{\Delta}^{2} u_{j}}{(\Delta x)^{2}} \int_{I_{j}} v_{1}^{2} d x
$$

yielding

$$
{\overline{\Delta^{2}}}_{j}=2(\Delta x)^{2} \frac{\int_{I_{j}}\left\{\left(x-x_{j}\right)^{2}-\frac{(\Delta x)^{2}}{12}\right\} u d x}{\int_{I_{j}}\left\{\left(x-x_{j}\right)^{2}-\frac{(\Delta x)^{2}}{12}\right\}^{2} d x}=\frac{360}{(\Delta x)^{2}} \int_{I_{j}}\left\{\left(x-x_{j}\right)^{2}-\frac{(\Delta x)^{2}}{12}\right\} u d x .
$$

We will defer details of this Optimal Recovery scheme and its analysis to a future journal paper. The eigenvalues of the scheme's $3 \times 3$ matrix operator can be obtained with a cubicequation solver and are displayed in Figure 3. All three are negative real, as required for stability. The good eigenvalue again is called $\lambda_{1}$; from Figure 4 it can be seen that the relative error in $\lambda_{1}$ is, unexpectedely, of the eighth order:

$$
\lambda_{1}=-\beta^{2}+O\left(\beta^{10}\right) .
$$

Thus, the scheme has an eigenfunction that represents the diffusion operator with eighth-order accuracy. It turns out that the two bad eigenfunctions, corresponding to $\lambda_{2}$ and $\lambda_{3}$, occur only 


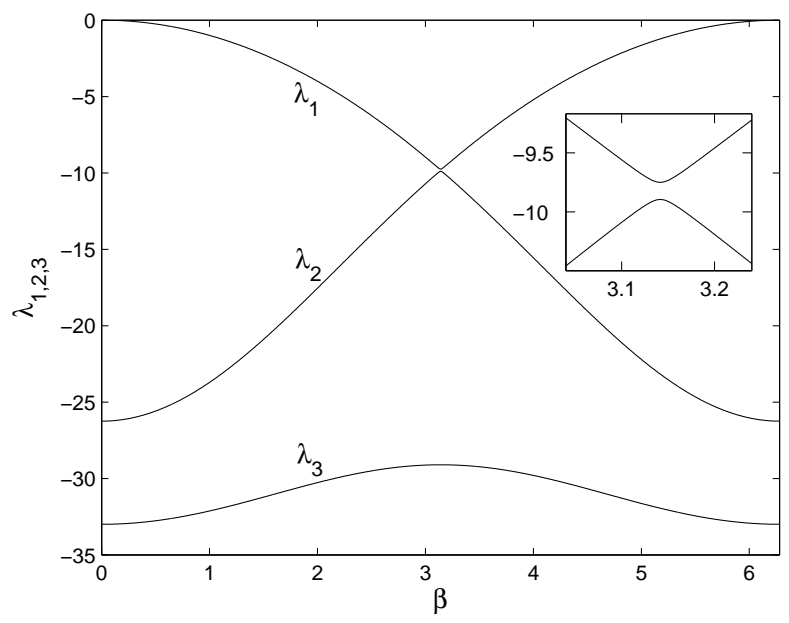

Figure 3: Eigenvalues $\lambda_{1,2,3}$ as functions of $\beta$ for the Optimal Recovery scheme with $p=2$. All three are negative. The insert shows the behavior of $\lambda_{1}$ and $\lambda_{2}$ near $\beta=\pi$, indicating that the two curves do not cross each other.

with amplitude $O\left(\beta^{8}\right)$ in the discretized initial values (see Appendix A), so the Optimal Recovery scheme for $p=2$ may be eighth-order accurate, at least when regarding only the cell averages $\bar{u}_{j}$. At the time of submission of this paper we had not been able to confirm this better-than-expected accuracy, because the scheme produced error norms at round-off level even on the coarsest grids.

\section{How does recovery fit in?}

\subsection{Optimal recovery}

The question immediately arises whether the Recovery scheme (77) fits into the family (32) of $(\sigma, \mu)$-schemes. The answer is negative. By choosing $\mu=9 / 4, \sigma=-1$ one can make most entries in the matrix $M(\sigma, \mu)$ equal to those in $M_{r e c}$, except the term proportional to $T-2 I+T^{-1}$ in the $(2,2)$ element. It bears the coefficient 3 , independent of $\sigma$ and $\mu$; the value in $M_{\text {rec }}$ is 2 .

Earlier it was stated without proof that for $p=1$ the choice $\mu=\frac{9}{4}$ combined with $\sigma=\frac{1}{4}$ yields a fourth-order accurate scheme. For higher values of $p$, however, new bilinear terms with $p-1$ extra parameters would have to be introduced in order to achieve the maximum possible accuracy.

\subsection{Suboptimal recovery}

Since the DG scheme for $p=1$ requires only a reliable recovery of $u$ and $u_{x}$ at the interfaces, one has the freedom to take the recovery of $u_{x x}$ and $u_{x x x}$ less seriously. One way to modify the recovery is by a kind of post-processing, namely, by averaging $f$ over an interval centered on the interface; this ensures that the reconstruction remains directionally unbiased. If we make the interval extend from $\xi=-\frac{\Delta x}{2}$ to $\xi=\frac{\Delta x}{2}$ (the staggered cell), the following averaged quantities 


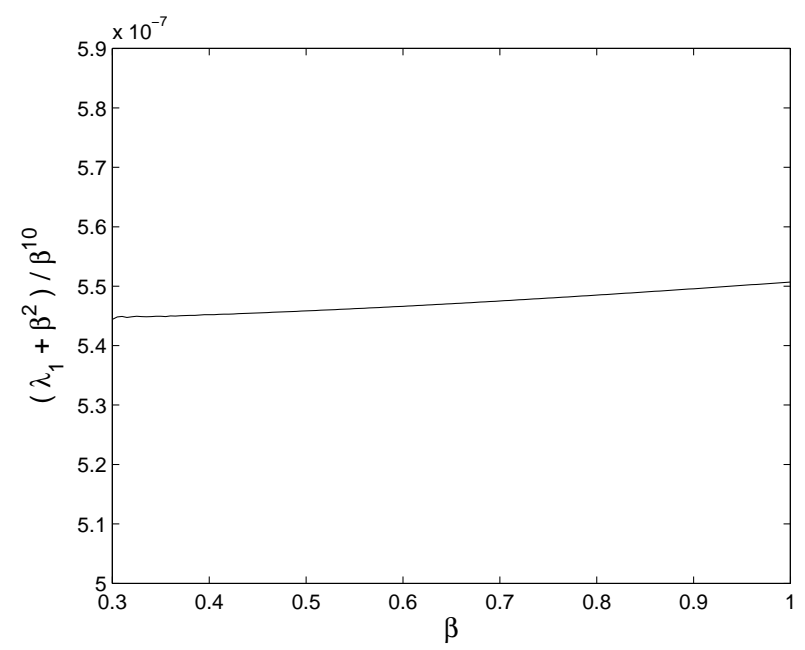

Figure 4: Error in the most accurate eigenvalue $\lambda_{1}$ as a function of $\beta$, normalized by $\beta^{10}$, for the Optimal Recovery scheme with $p=2$.

are obtained:

$$
\begin{gathered}
\bar{f}_{0}=\frac{1}{\Delta x} \int_{-\frac{\Delta x}{2}}^{\frac{\Delta x}{2}} f d \xi=f_{0}+\frac{(\Delta x)^{2}}{24} f_{0}^{\prime \prime}, \\
{\overline{f^{\prime}}}_{0}=\frac{1}{\Delta x} \int_{-\frac{\Delta x}{2}}^{\frac{\Delta x}{2}} f^{\prime} d \xi=f_{0}^{\prime}+\frac{(\Delta x)^{2}}{24} f_{0}^{\prime \prime \prime} .
\end{gathered}
$$

When inserting these averaged quantities into scheme (77) instead of the point values $f_{0}$ and $f_{0}^{\prime}$, the scheme becomes

$$
\frac{\partial}{\partial t}\left(\begin{array}{c}
\bar{u}_{j} \\
\overline{\Delta u}_{j}
\end{array}\right)=\frac{D}{(\Delta x)^{2}}\left(\begin{array}{cc}
\frac{13}{8}\left(T-2 I+T^{-1}\right) & -\frac{5}{16}\left(T-T^{-1}\right) \\
\frac{15}{4}\left(T-T^{-1}\right) & \left(T-2 I+T^{-1}\right)-\frac{15}{8}\left(T+2 I+T^{-1}\right)
\end{array}\right)\left(\begin{array}{c}
\bar{u}_{j} \\
\overline{\Delta u}_{j}
\end{array}\right)
$$

Again, this scheme is not one of the $(\sigma, \mu)$-family (32). Note that the update equation for $\overline{\Delta u}_{j}$ is no longer consistent with the third-order PDE (56).

The Fourier transform of the above matrix operator, $M_{r e c, s m}$, is given by

$$
\hat{M}_{r e c, s m}(\beta)=\left(\begin{array}{cc}
-\frac{13}{4}(1-\cos \beta) & -\frac{5}{8} i \sin \beta \\
\frac{15}{2} i \sin \beta & -2(1-\cos \beta)-\frac{15}{4}(1+\cos \beta)
\end{array}\right)
$$

and its eigenvalues are

$$
\begin{aligned}
& \lambda_{1}=-2(1-\cos \beta)=-\beta^{2}+O\left(\beta^{4}\right), \\
& \lambda_{2}=-7-\frac{1}{2} \cos \beta .
\end{aligned}
$$


Just as for the Stabilized Symmetric scheme $(\sigma=-1, \mu=1)$, the accurate eigenvalue is exactly the value found in the piecewise constant case $p=0$, i. e., for scheme (35). Apparently, the smoothing of the reconstruction over the staggered cell has produced a discretization equivalent to the simplest consistent discretization of the diffusion operator.

\subsection{Extending the DG diffusion family}

That terms in the recovery-based schemes are not accounted for in the $(\sigma, \mu)$-family indicates the family needs to be extended. This can be done by including more interface terms on the right-hand side of Eqn. (32).

After inspecting various possibilities we found one term that has the desired effect for $p=1$ while having no effect for $p=0$ :

$$
\mathcal{T}(T, \omega)=\omega D \Delta x\left(\left.\left[v_{x}\right]\left[u_{x}\right]\right|_{j+\frac{1}{2}}+\left.\left[v_{x}\right]\left[u_{x}\right]\right|_{j-\frac{1}{2}}\right) .
$$

Its sole contribution is to add $-12 \omega\left(T-2 I+T^{-1}\right)$ to the $(2,2)$ element of the matrix operator $M(T, \sigma, \mu)$ given by Eqn. (41). The three-parameter scheme becomes

$$
\frac{\partial}{\partial t}\left(\begin{array}{c}
\bar{u}_{j} \\
\overline{\Delta u}_{j}
\end{array}\right)=\frac{D}{(\Delta x)^{2}} M(T, \sigma, \mu, \omega)\left(\begin{array}{c}
\bar{u}_{j} \\
\overline{\Delta u}_{j}
\end{array}\right)
$$

with

$$
M(T, \sigma, \mu, \omega)=\left(\begin{array}{cc}
\mu\left(T-2 I+T^{-1}\right) & \frac{1-\mu}{2}\left(T-T^{-1}\right) \\
6(\sigma+\mu)\left(T-T^{-1}\right) & (3-12 \omega)\left(T-2 I+T^{-1}\right)-3(\sigma+\mu)\left(T+2 I+T^{-1}\right)
\end{array}\right) .
$$

Hence, by choosing

$$
\begin{aligned}
\mu & =\frac{9}{4} \\
\sigma & =-1 \\
\omega & =\frac{1}{12}
\end{aligned}
$$

the fourth-order-accurate Recovery scheme (77) reappears. The suboptimal Recovery scheme (88) is found by choosing

$$
\begin{aligned}
\mu & =\frac{13}{8}, \\
\sigma & =-1, \\
\omega & =\frac{1}{6} .
\end{aligned}
$$

Note that in both recovery-based schemes the value of $\sigma$ is -1 as in the Symmetric scheme; Baumann's choice, $\sigma=1$, appears to be an odd choice, unconnected to the recovery principle.

For $p \geq 2$ we expect more bilinear expressions and corresponding parameters to be needed in order to account for all terms in the higher-order Optimal Recovery schemes. 


\section{A numerical comparison}

We pitted the Optimal Recovery scheme against the members of the $(\sigma, \mu)$-family discussed above (Stabilized Symmetric, Baumann and fourth-order schemes) and the Suboptimal Recovery scheme, in a simple 1-D numerical test. The schemes were applied to an initial/boundary-value problem for the Poisson equation

$$
u_{t}=D u_{x x}+4 \pi^{2} D \sin (2 \pi x)
$$

subject to the initial and boundary values

$$
\begin{aligned}
u(x, 0) & =\cos (2 \pi x), \quad x \in(0,1) \\
u(0, t) & =1, \quad t \geq 0 \\
u_{x}(1, t) & =2 \pi-1, \quad t \geq 0
\end{aligned}
$$

Only the accuracy of the final steady solution,

$$
u(x, \infty)=\sin (2 \pi x)+1-x
$$

was considered. On a sequence of grids the exact cell averages of the steady solution were compared to those obtained by the different schemes

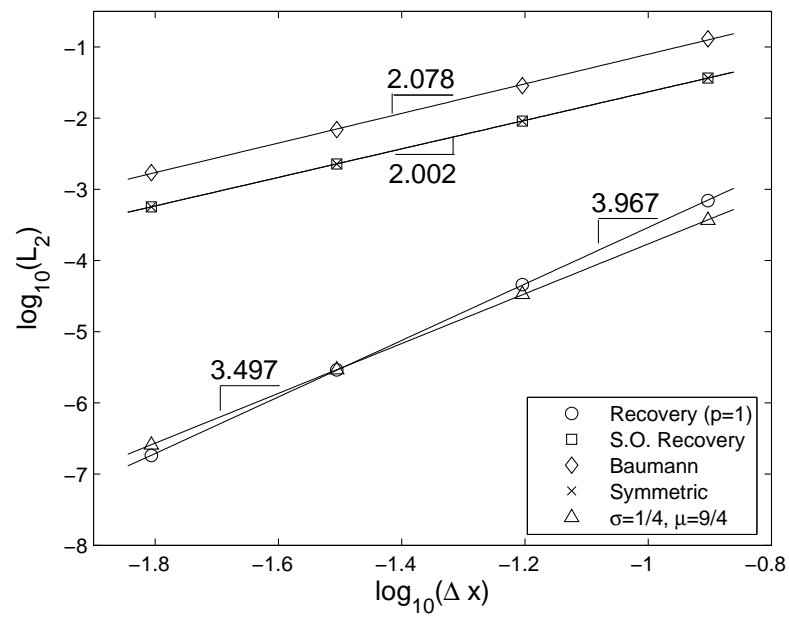

Figure 5: $L_{2}$ error norms for the Recovery, Suboptimal Recovery, Symmetric, Baumann and $(1 / 4,9 / 4)$ schemes, for $p=1$.

The cell average of the source term needed in the schemes was always computed analytically. At the Dirichlet and Neumann boundaries, second-order and fourth-order recovery, based on the boundary condition and the data from one or two interior cells, were tested for the Recovery scheme.

Figure 5 shows the $L_{2}$ errors for $p=1$ for the four schemes studied, as a function of the cell size. The $L_{1}$ and $L_{\infty}$ errors (not shown) behave similarly, with one exception. The Optimal 
Recovery scheme with fourth-order recovery at the boundaries is seen to achieve fourth-order accuracy. The scheme with $\sigma=\frac{1}{4}, \mu=\frac{9}{4}$ has order 3.5 in $L_{2}$; its order varies from 4 in $L_{1}$ (not shown) to 3 in $L_{\infty}$ (not shown). This suggests the presence of a third-order boundary error that could still be reduced, for instance, by using recovery solely at the boundary.

The Suboptimal Recovery scheme and Stabilized Symmetric scheme show their second-order accuracy and equal error level. The Baumann scheme is also second-order accurate, but has a larger error. In this test case the Neumann condition appears to damp out the the $2 \Delta x$-mode the Baumann scheme itself can't get rid of. Switching to a Dirichlet condition that produces the same steady solution does generate the odd-even grid-decoupling, as seen in Figure 6. For the amplitude of the mode it made no difference whether the grid consisted of an even or odd number of cells.

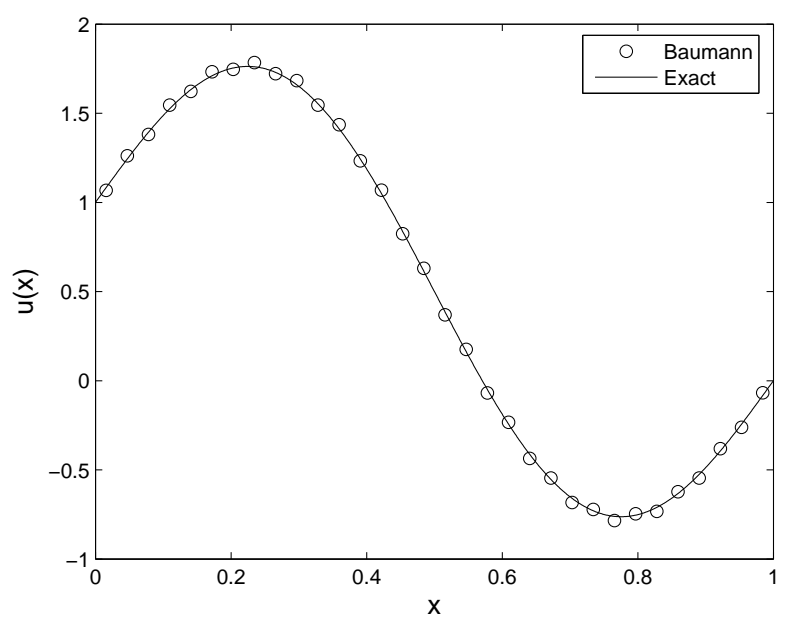

Figure 6: Numerical solution produced by Baumann's scheme (symbols), plotted over the exact steady state solution (solid line). Dirichlet conditions at both boundaries. Note the odd-even decoupling.

Figure 6 shows the numerical solution (cell averages) produced by Baumann's scheme on a grid of 32 cells, with Dirichlet conditions at both boundaries. The odd-even decoupling is clearly visible.

Figure 7 shows that the accuracy of the Optimal recovery scheme is compromised if quadratic rather than quartic reconstruction is used at the boundaries. Plotted is the $L_{\infty}$ norm, which shows the accuracy reduction most clearly.

We also solved the test problem using the Optimal Recovery scheme with $p=2$, i. e., with piecewise parabolic subcell distributions. Originally we expected the order of accuracy of the scheme to be $6(=2 p+2)$, with the norms going down as $(\Delta x)^{6}$, but the eigenvalue and eigenvector analysis, as reported in this paper, suggests the order of accuracy of the scheme may be $8\left(=p^{2}+p+2 ?\right)$. At the time of submission of this paper we had not yet confirmed this in a grid-refinement study, as the error norms for a 4-cell grid already had dropped to the rounding-error level. The question of the scheme's order therefore remains open. 


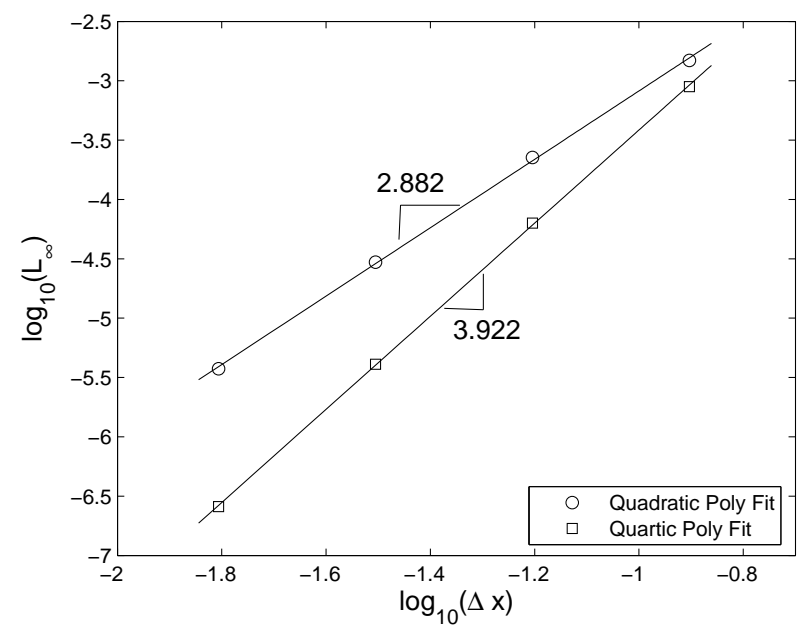

Figure 7: Effect of the degree of recovery (polynomial fitting) at the boundaries on the order of accuracy of the Optimal Recovery scheme with $p=1$. The effect is best seen in the convergence of the $L_{\infty}$ norm, shown here.

\section{Conclusions and perspective}

In this paper we have created DG schemes for the diffusion equation using at each interface a local recovery of the underlying smooth solution from the discontinuous discrete solution. Recovered solution, smooth solution and discrete solution are all equal in a weak sense. This is a literal way of removing the discontinuities in the weak sense, as opposed, for example, to adding a finite-element-style penalty term.

Before opting for recovery, much attention has been given to the fact that advection and diffusion schemes are fundamentally different. It is argued that recovery of the diffusive fluxes is not in contradiction with upwinding of the advective fluxes.

When the recovery is done with maximum accuracy, in an example based on a piecewise linear discrete solution, a fourth-order-accurate scheme results. Dropping the accuracy of the recovery by a cell-averaging procedure reduces the accuracy to that of the scheme based on a piecewise constant solution. Neither scheme is included in the standard family of DG schemes. Using a piecewise quadratic basis seems to make the scheme eigth-order accurate; this result remains to be confirmed by a numerical test.

We have indicated that the family can be extended so as to include the recovery-based schemes, by adding a boundary term previously ignored in finite-element theory.

We challenge the finite-element community to come up with its own way of describing these Recovery schemes, e. g., by generating bilinear terms as Lagrange multipliers in a variational principle. This may not be easy, given the limited number of bilinear forms that so far have been judged useful; the task will only get harder when increasing the order of the solution representation. The recovery principle will give guidance in separating those choices that make physical sense from those that don't.

Meanwhile, the recovery principle produces excellent diffusion schemes, and may be extended 
to multiple dimensions and unstructured grids by any means.

\section{Acknowledgements}

The first author is indebted to Marc van Raalte (CWI, Amsterdam, Netherlands) for his illuminating tutorials on the state of the art in DG methods for diffusion. The first author was supported in part by the US Department of Defense, Department of the Air Force, Award Nr. F49620-03-1-0226, and by the Theme MAS-2 at the CWI, Amsterdam, Netherlands.

\section{Appendix A: Good and bad eigenfunctions}

\section{A1 Initial-value approximation}

When determining the formal accuracy of a DG scheme, two kinds of errors need to be considered:

- the error in approximating the differential operator - for this we inspect the most accurate eigenvalue;

- the error in projecting the true initial values onto the basis funtions - for this we must inspect the eigenfunctions.

The first error determines the accuracy of any steady solution, and puts a lower limit on the evolutionary error when the DG operator is combined with a suitable time-marching scheme. The projection error is a starting error $^{8}$ : it is the deviation of the accurate eigenfunction (belonging to the accurate eigenvalue) from the piecewise continuous discretized initial values. This deviation is in the form of the other eigenfunctions, corresponding to the inaccurate eigenvalue(s), which are inconsistent with the diffusion operator. The two kinds of errors are not necessarily of the same order.

Below are determined the projection errors of some of the key DG diffusion schemes based on a piecewise linear solution representation $(p=1)$. To set the stage, the initial-value discretization first has to be analyzed. Assumem therefore, that the exact initial values form a Fourier mode,

$$
U(x)=U_{0} e^{i \beta x / \Delta x}
$$

discretization in cell $j$ yields

$$
\begin{aligned}
\bar{u}_{j} & =\frac{1}{\Delta x} \int_{I_{j}} U(x) d x=\frac{\sin (\beta / 2)}{\beta / 2} U_{j}, \\
\overline{\Delta u}_{j} & =\Delta x \frac{\int_{I_{j}}\left(x-x_{j}\right) U(x) d x}{\int_{I_{j}}\left(x-x_{j}\right)^{2} d x}=\frac{12 i}{\beta}\left(\frac{\sin (\beta / 2)}{\beta / 2}-\cos (\beta / 2)\right) U_{j} .
\end{aligned}
$$

Correspondingly, in accordance with Eqn. (37) the initial values (107) are approximated by

$$
u(x)=\left\{\frac{\sin (\beta / 2)}{\beta / 2}+\frac{x-x_{j}}{\Delta x} \frac{12 i}{\beta}\left(\frac{\sin (\beta / 2)}{\beta / 2}-\cos (\beta / 2)\right)\right\} U_{j}, \quad x \in I_{j} .
$$

For small $\beta$ the gradient of this function tends to $i \frac{\beta}{\Delta x} U_{j}$, consistent with Eqn. (49).

\footnotetext{
${ }^{8} \mathrm{At}$ least, for a linear operator; in a nonlinear scheme this error is committed at every time step because the bad eigenfunction keeps getting regenerated.
} 


\section{A2 Optimal Recovery scheme with lineear basis}

We shall first investigate the optimal Recovery scheme, with eigenvalues $\lambda_{1}$ and $\lambda_{2}$ given by (79). The corresponding eigenvectors, conveniently normalized, are

$$
\begin{aligned}
& \mathbf{r}_{1}=\left(\begin{array}{c}
\frac{2}{\beta} \sin \frac{\beta}{2} \\
-\frac{6 i}{\beta \cos (\beta / 2)}\left\{1-\frac{8}{15}(1-\cos \beta)-\sqrt{1-\frac{2}{5}(1-\cos \beta)-\frac{11}{225}(1-\cos \beta)^{2}}\right\}
\end{array}\right) \\
& \mathbf{r}_{2}=\left(\begin{array}{c}
\frac{2}{\beta} \sin \frac{\beta}{2} \\
-\frac{6 i}{\beta \cos (\beta / 2)}\left\{1-\frac{8}{15}(1-\cos \beta)+\sqrt{1-\frac{2}{5}(1-\cos \beta)-\frac{11}{225}(1-\cos \beta)^{2}}\right\}
\end{array}\right) .
\end{aligned}
$$

These eigenvectors in turn define linear eigenfunctions in each cell,

$$
\left.\begin{array}{l}
g_{1}(x)=r_{11}+\frac{r_{12}}{\Delta x}\left(x-x_{j}\right) \\
g_{2}(x)=r_{21}+\frac{r_{22}}{\Delta x}\left(x-x_{j}\right)
\end{array}\right\} \quad x \in I_{j}
$$

from which the discrete solution (110) can be built up. Note that both eigenfunctions have been scaled such that their cell average matches that of the discrete solution; the distinction is in the gradients. For small $\beta$ the gradient $r_{12} / \Delta x$ of the first eigenfunction tends to $i \beta / \Delta x$, showing it is close to the discrete solution. The gradient of the second eigenfunction tends to $-24 i /(\beta \Delta x)$, completely off the map.

Regarding accuracy, the key question is now how much of $g_{2}(x)$ is needed to compose $u(x)$. Writing

$$
u(x)=C_{1} g_{1}(x)+C_{2} g_{2}(x)
$$

one easily finds

$$
\begin{gathered}
C_{1}+C_{2}=1, \\
-\left(C_{1}+C_{2}\right)\left\{1-\frac{8}{15}(1-\cos \beta)\right\} \\
\left(C_{1}-C_{2}\right) \sqrt{1-\frac{2}{5}(1-\cos \beta)-\frac{11}{225}(1-\cos \beta)^{2}}= \\
2 \cos (\beta / 2)\left(\frac{\sin (\beta / 2)}{\beta / 2}-\cos (\beta / 2)\right),
\end{gathered}
$$

from which the following value for $C_{2}$ emerges:

$$
C_{2}=\frac{\cos (\beta / 2)}{2}\left(1-\frac{2 \frac{\sin \beta}{\beta}-1+\frac{7}{15}(1-\cos \beta)}{\sqrt{1-\frac{2}{5}(1-\cos \beta)-\frac{11}{225}(1-\cos \beta)^{2}}}\right) .
$$

For small $\beta$ we find

$$
C_{2}=O\left(\beta^{6}\right)
$$




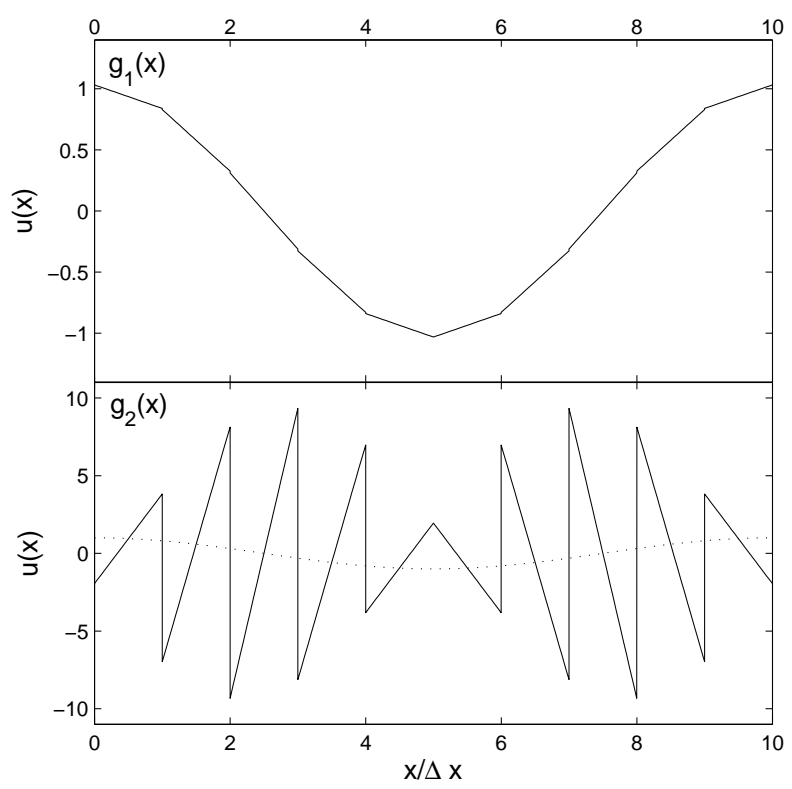

Figure 8: Piecewise linear approximation of the function $\cos (\beta x / \Delta x)$, with $\beta=\pi / 5$, by the eigenfunctions of the Optimal Recovery scheme with $p=1$. Top: using the eigenfunction $g_{1}$; bottom: using the eigenfunction $g_{2}$. Note the different scaling of $u$.

Thus, the initial-value projection onto $g_{1}$ is sixth-order accurate. As the approximation of the diffusion operator based on this eigenfunction is fourth-order accurate (see Eqn. (80)), the Recovery scheme is a genuine fourth-order scheme. Note that the abysmal gradient value in $g_{2}$, when multiplied by $\beta^{6}$, causes an $O\left(\beta^{4}\right)$ relative starting error in the subcell solution gradient.

To illustrate the above formulas and analysis, Figure 8 shows the real part of a Fourier mode with a wavelength of $10 \Delta x$, as represented by the first eigenfunction (top) and by the second eigenfunction (bottom); the sequence of cell averages is identical in both graphs. The bad representation shows subcell gradients that are more than an order of magnitude too large and have the wrong sign. Figure 9 shows that the amplitude of the second eigenfunction in the discretized Fourier mode is only $O\left(\beta^{6}\right)$.

For comparison, Figure 10 shows the representation of the same Fourier mode by the eigenfunctions of the DG advection scheme for $p=1$, obtained almost 30 years ago [6]. Although the formulas for the eigenvalues and eigenvectors are different from those for the diffusion scheme, the eigenfunctions are indistinguishable within the "eyeball norm". This suggests that the widely different advection and diffusion approaches are very compatible, and that the eigenfunctions for a convection-diffusion DG scheme will be very similar.

\section{A3 Other linear-basis schemes}

Without proof we present some results regarding schemes of the $(\sigma, \mu)$-family, for $p=1$. Details are deferred to a future journal paper. 


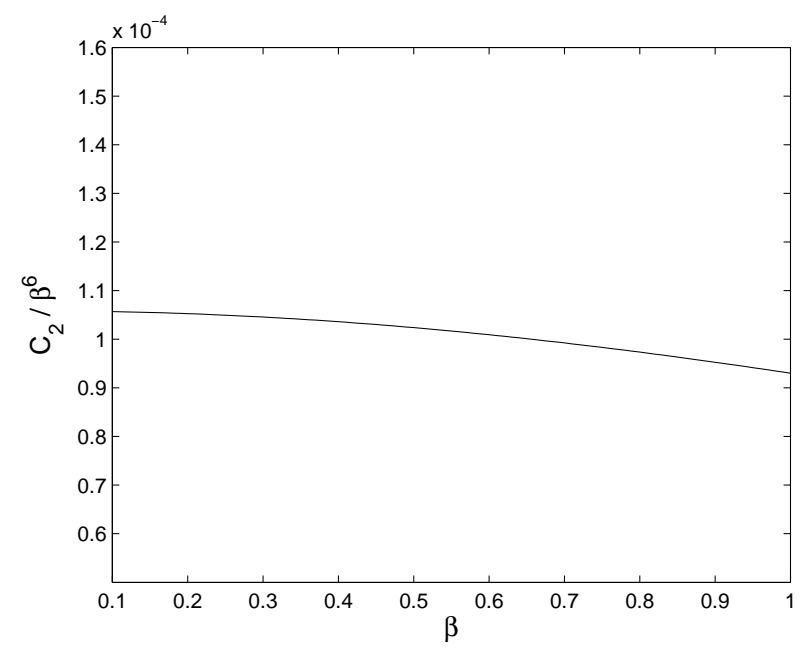

Figure 9: Amplitude $C_{2}$ of the second eigenfunction $g_{2}$ as a function of $\beta$, normalized by $\beta^{6}$, for the Optimal Recovery scheme with $p=1$.

Baumann's scheme, with $\sigma=1, \mu=0$, can be shown to have a fourth-order starting error. The scheme is only second-order accurate as it approximates the diffusion operator with secondorder accuracy; cf. Eqn. (53).

The Stablized Symmetric scheme, with $\sigma=-1, \mu=1$, can be shown to have both secondorder projection and operator errors.

Earlier it was stated without proof that satisfying (57) is sufficient for making the gradient update consistent with the gradient-evolution equation (56). Among the resulting one-parameter family of schemes there is one with a fourth-order accurate eigenvalue, viz., for $\mu=\frac{9}{4}$; its bad eigenfunction appears with weight $O\left(\beta^{4}\right)$, so the scheme is a true fourth-order accurate scheme.

\section{A4 Optimal Recovery scheme with quadratic basis}

Details of the eigenvector analysis for the Optimal Recovery scheme with $p=2$ are deferred to a future journal paper; its results are summarized in the last set of figures.

Figure 11 shows the real part of a Fourier mode with a wavelength of $20 \Delta x$, as represented by the first (top), second (middle) and third eigenfunction (bottom); the sequence of cell averages is identical in all three graphs. The range of the third eigenfunction is two orders of magnitude larger than that of the approximated Fourier mode.

Figure 12 shows that the amplitudes $C_{2}$ and $C_{3}$ of the two bad eigenfunctions in a discretized Fourier mode are only $O\left(\beta^{8}\right)$. It is not yet clear if the order of accuracy of the overall scheme is 8 or, as expected, $6(=2 p+2)$.

\section{References}

[1] B. Cockburn, "Discontinuous Galerkin methods for convection-dominated problems," in High-Order Methods for Computational Physics (T. J. Barth and H. Deconcinck, eds.), 


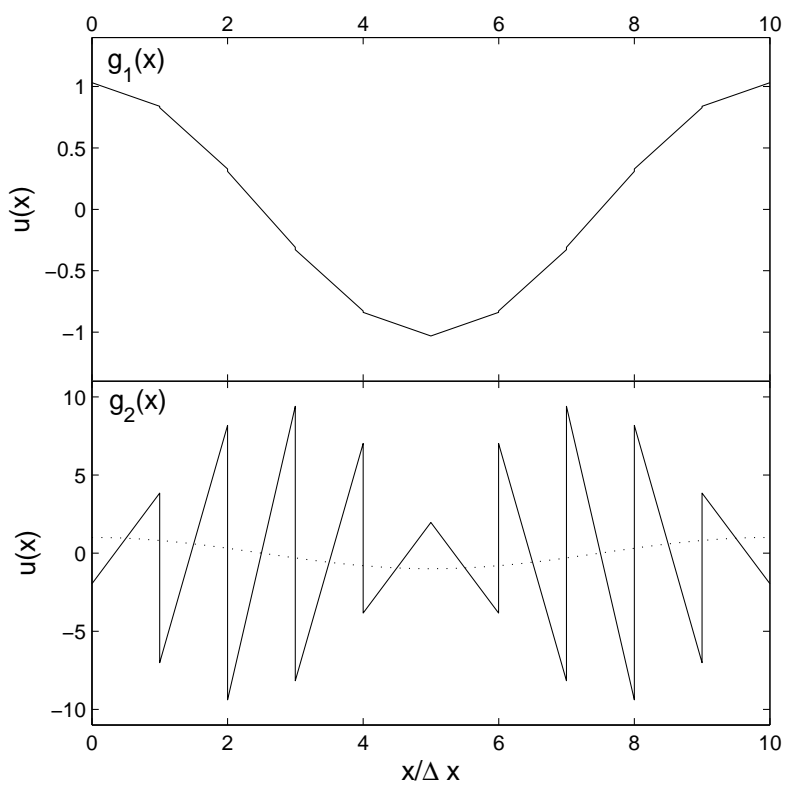

Figure 10: Piecewise linear approximation of the function $\cos (\beta x / \Delta x)$ with $\beta=\pi / 5$ by the eigenfunctions of the DG scheme with $p=1$ for advection. Top: using the accurate eigenfunction; bottom: using the bad eigenfunction. Note the different scaling of $u$.

vol. 9, pp. 69-224, Springer, New York, 1999.

[2] R. B. Lowrie and J. E. Morel, "Methods for hyperbolic systems with stiff relaxation," International Journal for Numerical Methods in Fluids, vol. 40, pp. 413-423, 2002.

[3] T. Oliver, K. Fidkowski, and D. L. Darmofal, "Multigrid solution for high-order Discontinuous Galerkin discretizations of the compressible Navier-Stokes equations." Presented at the 3rd International Conference on Computational Fluid Dynamics, June 12-16, 2004, Toronto, ON, Canada, 2004.

[4] Y. Suzuki, B. van Leer, and J. A. F. Hittinger, "Investigation of the Discontinuous Galerkin method for first-order PDE approaches to CFD," 2005. Extended Abstract submitted to 17th AIAA Computational Fluid Dynamics Conference, 6-9 June 2005, Toronto, ON, Canada.

[5] W. Reed and T. Hill, "Triangular mesh methods for the neutron transport equation," Tech. Rep. LA-UR 73-479, Los Alamos National Laboratory, 1973.

[6] B. van Leer, "Towards the ultimate conservative difference scheme. IV. A new approach to numerical convection," Journal of Computational Physics, vol. 23, pp. 276-299, 1977.

[7] D. N. Arnold, "An interior penalty finite element method with discontinuous elements," SIAM Journal on Numerical Analysis, vol. 19, pp. 742-760, 1982. 


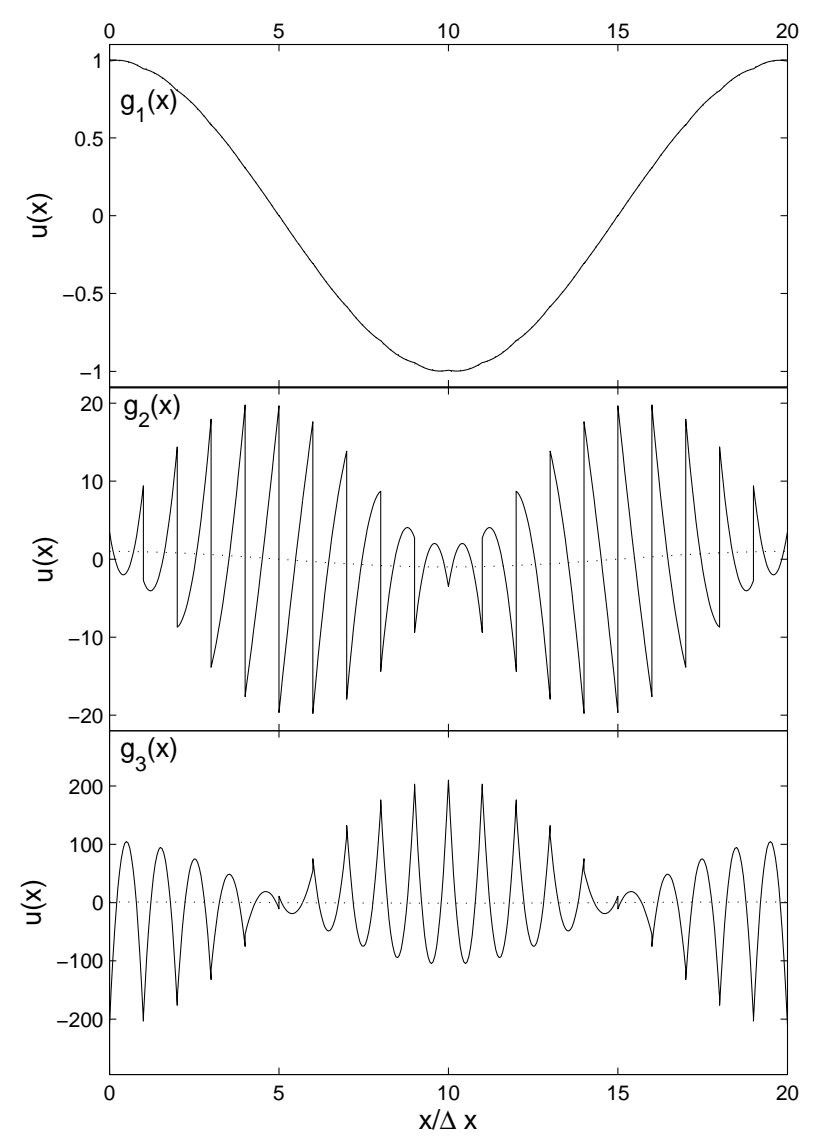

Figure 11: Piecewise quadratic approximation of the function $\cos (\beta x / \Delta x)$ with $\beta=\pi / 5$ by the eigenfunctions of the Optimal Recovery scheme with $p=2$. Top: using the most accurate eigenfunction $g_{1}$; middle: using the second eigenfunction $g_{2}$; bottom: using the third eigenfunction $g_{3}$. Note the different scaling of $u$.

[8] C. E. Baumann, An hp-adaptive discontinuous Finite Element Method for Computational Fluid Dynamics. PhD thesis, University of Texas at Austin, 1997.

[9] J. T. Oden, I. Babuska, and C. E. Baumann, "A discontinuous $h p$-finite element method for diffusion problems," Journal of Computational Physics, vol. 146, pp. 491-519, 1998.

[10] M. H. van Raalte, Multigrid Analysis and Embedded Boundary Conditions for Discontinuous Galerkin Discretization. PhD thesis, University of Amsterdam, 2004.

[11] D. N. Arnold, F. Brezzi, B. Cockburn, and L. D. Marini, "Unified analysis of Discontinuous Galerkin methods for elliptic problems," SIAM Journal on Numerical Analysis, vol. 39, pp. 1749-1779, 2002.

[12] J. E. Fromm, "A method for reducing dispersion in convective difference schemes," Journal of Computational Physics, vol. 3, pp. 176-189, 1968. 


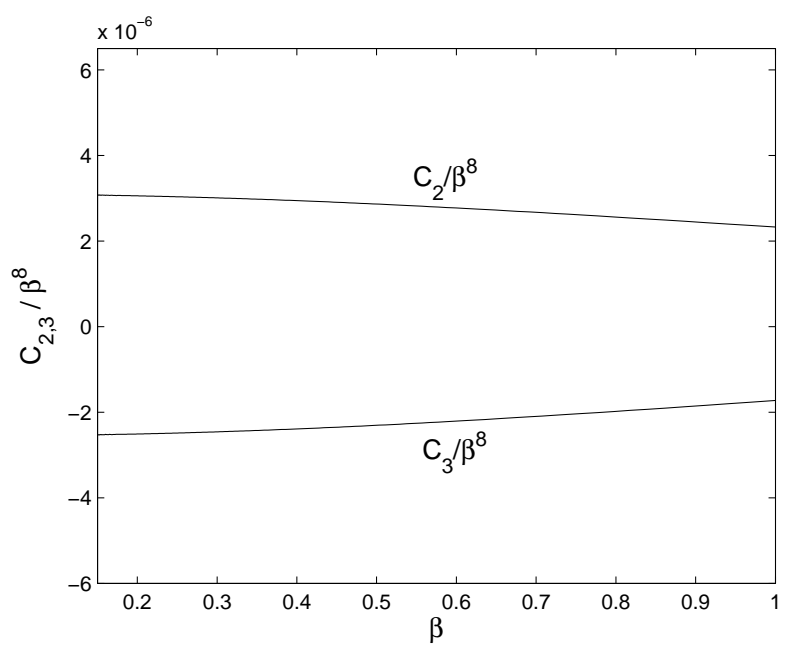

Figure 12: Amplitudes $C_{2}$ and $C_{3}$ of the two bad eigenfunctions for $\mathrm{p}=2$

[13] P. R. Woodward, "Piecewise-parabolic methods for astrophysical fluid dynamics," Tech. Rep. UCRL 90009, Lawrene Livermore National Laboratory, 1983.

[14] R. Lowrie, Compact Higher-Order Methods for Hyperbolic Conservation Laws. PhD thesis, University of Michigan, 1996.

[15] G. L. Russel and J. A. Lerner, "A new finite-differencing scheme for the tracer transport equation," Journal of Applied Meteorology, vol. 20, pp. 1483-1498, 1981.

[16] M. J. Prather, "Numerical advection by conservation of second-order moments," Journal of Geophysical Research, vol. 91, pp. 6671-6681, 1986.

[17] B. van Leer, "Towards the ultimate conservative difference scheme. V. A second-order sequel to Godunov's method," Journal of Computational Physics, vol. 32, pp. 101-136, 1979.

[18] L. F. Richardson, "The approximate arithmetical solution by finite differences of physical problems involving differential equations, with an application to the stresses in a masonry dam," Philosophical Transactions of the Royal Society of London, Series A, vol. 210, pp. 307$357,1910$. 